\title{
Is there an isolated arrhythmogenic right atrial myocarditis?
}

\author{
M. Fromer, C. Genton, * J. Schlaepfer, JJ. Goy, L. Kappenberger \\ Division of Cardiology, Medical Clinic, University Hospital, Lausanne and the *Institute of Pathology, \\ University of Lausanne, Switzerland
}

KEY WORDS: Atrial myocarditis, atrial tachycardia, endocardial resection.

Two cases with drug refractory ectopic atrial tachycardia are described. A map-guided partial resection of the right atrium $(R A)$ was done after preoperative endocardial catheter mapping had shown well-defined areas of fractionated RA potentials. Intraoperatively, there were no aneurysmal formations present as described by other authors. Histopathologic examination of the resected tissue showed atrial myocarditis in both patients. Postoperative right ventricular myocardial biopsies revealed no inflammatory tissue. A minor elevation of antibodies against echoviruses was found in one case. Postoperative electrophysiologic studies were negative. We conclude: focal $R A$ myocarditis without concomitant ventricular myocarditis may represent one cause of drug-resistant ectopic atrial tachycardia. Map-guided surgical intervention may cure the disease.

\section{Introduction}

The anatomic substrate of ectopic atrial tachycardia (AT) is rarely evaluated as the investigation of the underlying disease requires a surgical approach. Successful surgical resection was reported by a few groups ${ }^{[1-3]}$. Olsson et al. ${ }^{[1]}$ described an aneurysmal formation of the atrial tissue close to the right atrial appendix. Wyndham et al. ${ }^{[2]}$ reported in 1980 a case with patchy fibrosis of the right atrium close to the appendix, an increase of connective tissue and mononuclear cell infiltration. The same group reported later surgical experience with eight cases, however, the results of histologic examination were not given ${ }^{[3]}$. In our report of two cases we describe the occurrence of ectopic right AT without aneurysmal formation, however with welldefined inflammation of the right atrium in the absence of concomitant myocarditis of the right ventricle. We hypothesize that myocarditis of the right atrium may be responsible for recurrent ectopic AT resistant to antiarrhythmic drug regimens, and map-guided resection of the abnormal area may cure the disease.

Submitled for publication on 9 August 1989, and in revised form 26 September 1989.

Martın Fromer is recipient of a SCORE grant $\$ 3.750-87$ from the Suiss National Foundation of Sciences.

Address for Correspondence M. Fromer, MD. Division of Cardiology, Centre Hospitalier Universitaire Vaudois, CHUV, 1011 Lausanne, Suitzerland.

\section{Case 1}

A 20-year-old male was referred for electrophysiologic evaluation of a 1-year history of highly symptomatic, drug-resistant supraventricular tachycardia. Clinical and echocardiographic examination showed no associated cardiomyopathy. Standard techniques for electrophysiologic evaluation were used ${ }^{[4]}$. An atrial tachycardia with a cycle length of $300 \mathrm{~ms}$ and $2: 1$ atrioventricular conduction was present (see Fig. 1). Endocardial catheter mapping showed areas of abnormal electrical activity with fractionated potentials in the postero-lateral regions of the mid right atrium (Fig. 2). This area was considered to be responsible for the AT. This finding together with the patient's history motivated an attempt at surgical resection. Intraoperative epicardial mapping confirmed the presence of an ectopic right sided AT originating in the infero-posterior areas of the right atrium. The postoperative course was unremarkable and the atrial tachycardia did not reoccur.

\section{Case 2}

A 27-year-old male has been referred for the evaluation of a 5-year history of drug-resistant supraventricular tachycardia. Drug trials with disopyramide, verapamil, beta blockers, flecainide and propafenone were not effective. Clinical and echocardiographic evaluation disclosed no cardiovascular abnormality. The electrophysiologic study 

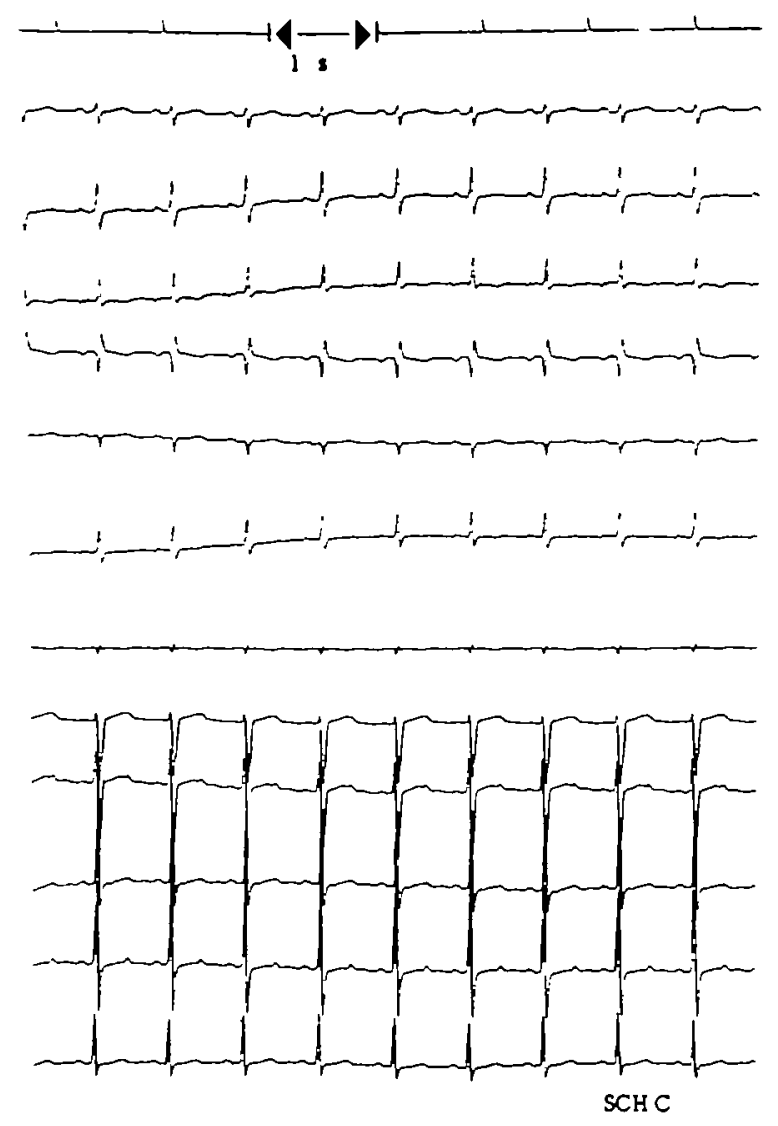

Figure I 12-lead standard electrocardiogram of patient 1 showIng an atrial tachycardia with 2:1 atrioventricular conduction. Paper speed $25 \mathrm{~mm} \mathrm{~s}^{-1}$.

showed an atrial tachycardia (Fig. 3) and large zones of fractionated potentials (Fig. 4) in the mid. right atrium. The same findings were reproducible during a second electrophysiologic study done preoperatively and motivated the surgical intervention. Epicardial mapping confirmed the presence of right sided AT. Intra-operatively, large zones with fragmented potentials were found around the appendix and anterolateral segments of the right atrium. A large resection including all areas of fragmented potentials was done and replaced with a pericardial patch. After this the AT was no longer inducible.

\section{Results of histologic examination}

CASE !

Six specimens were given for histologic examination. The largest measuring $2 \times 1 \times 0.5 \mathrm{~cm}$, the smallest $1.5 \times 0.7 \times 0.2 \mathrm{~cm}$. Histologically, a moderate inflammatory infiltrate was found in the myocardium and endocardium. This focally confluent infiltrate was essentially lymphocytic, there were no eosinophilic leucocytes nor giant cells. Obviously damaged myocytes with vacuolization were present (Fig. 5). In some areas, granulation tissue with the beginning of fibrosis was present. There were no mural thrombi. These findings are consistent with a moderate, diffuse lymphocytic myocarditis with granulation tissue and fibrosis. Eight days later, right ventricular endomyocardial biopsies were obtained from the lateral wall and septum. Examination of multiple sections of the six specimens suggested an increased interstitial cellularity but immunohistochemical staining for polymorphonuclear leucocytes and lymphocytes were negative. The specimens were therefore considered to represent normal myocardium. 


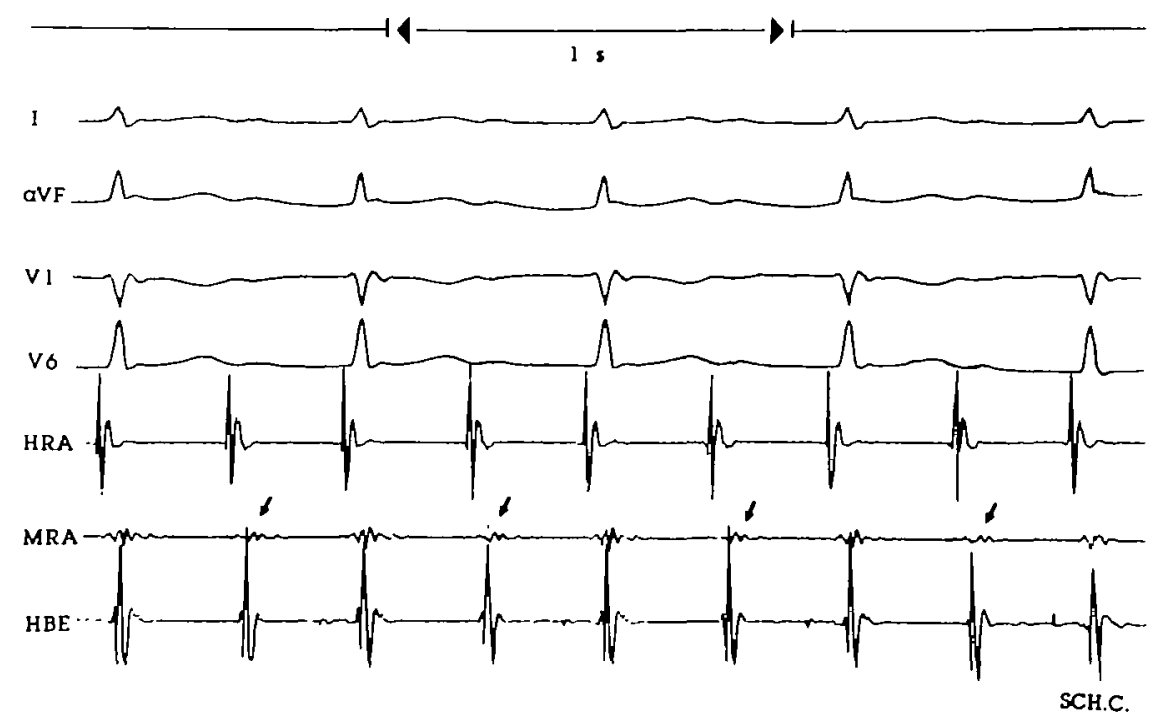

Figure 2 Representative illustration of local recordings of right atrial electrocardiograms in patient I durng atrial tachycardia with 2:1 AV-conduction. Arrows indicate nght atrial potentrals with abnormal low amplitude. Note for comparison the normal right atrial potentials (HRA) with rapid rise of the intrnnsic deflection and high amplitude of the local electrocardiogram and shorter duration of the signal. I, a VF, VI and V6 represent standard surface electrocardiographic leads. Other abbreviations: $\mathrm{HRA}=$ high right atrium, $\mathrm{MRA}=$ mid right atruum, $\mathrm{HBE}=\mathrm{His}$ bundle electrocardiogram, including low right atrial depolarization. Paper speed $100 \mathrm{~mm} \mathrm{~s}^{-1}$.

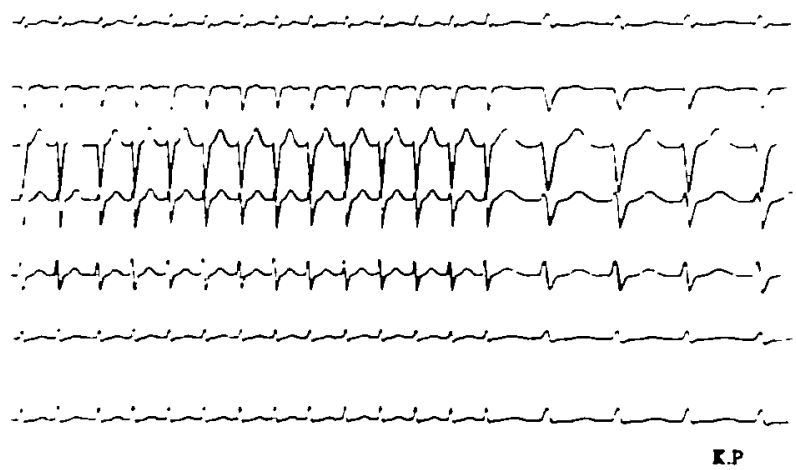

Figure 3 12-lead electrocardiagrams of patient 2 showing ectopic right atrial tachycardia with $1: 1$ conduction. Paper speed at left

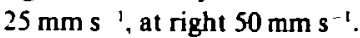




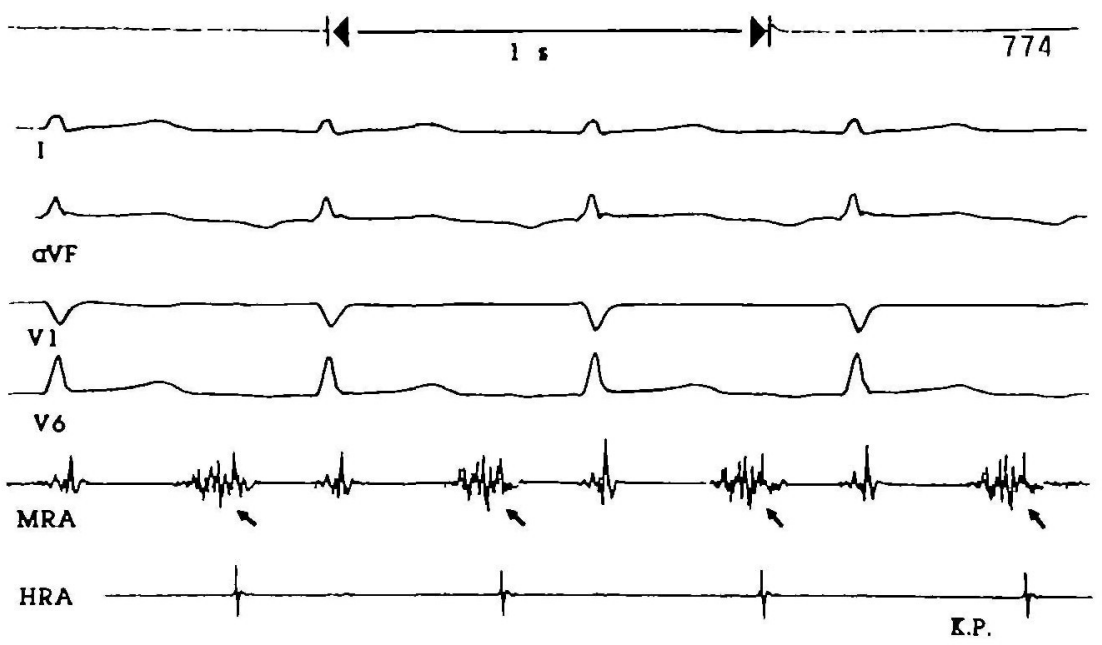

Figure 4 Local recordings of right atrial electrocardiograms in patient 2 during right atral tachycardia with 1:1 conduction. Arrows indicate abnormal fragmented nght atrial potentials as discussed in Fig. 2. For abbreviations see Fig. 2.

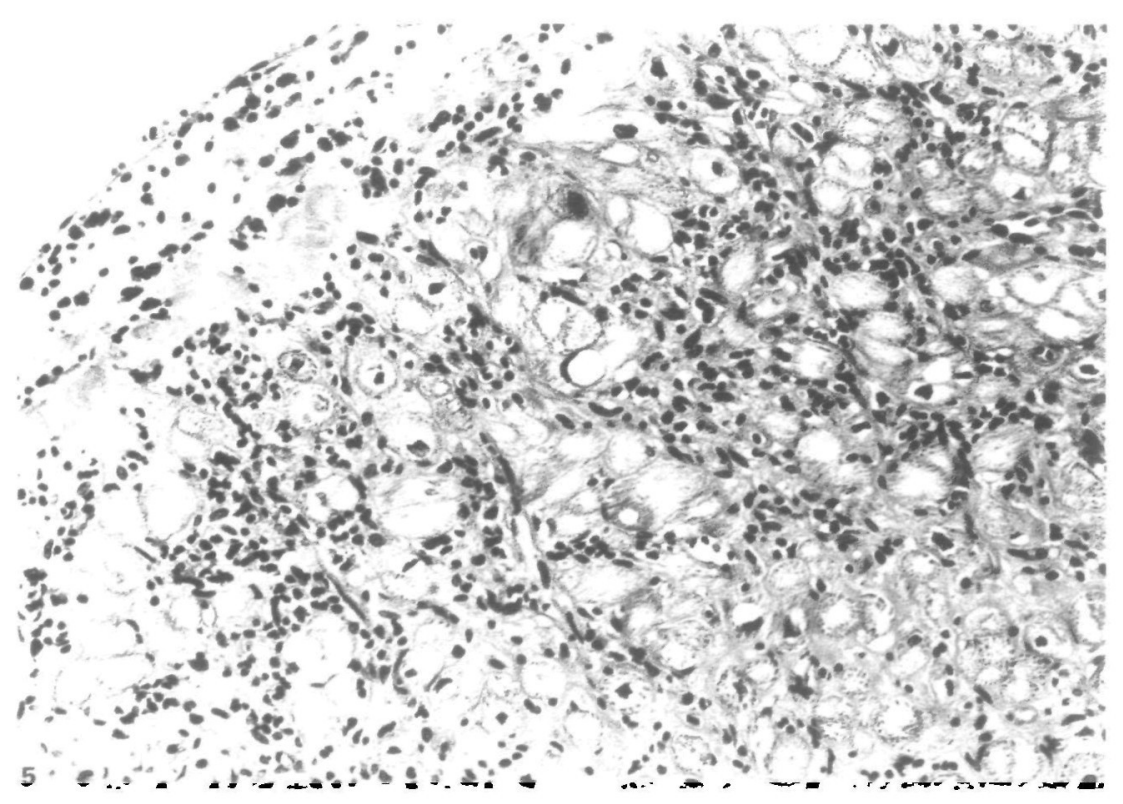

Figure 5 Confluent lymphocytic infiltrate of myocardium and endocardium accompanied by obvious vacuolization of myocytes and beginning fibrosis. Haematoxylin and eosin $\times 250$.

\section{CASE 2}

The resected part of the right atrium measured $6 \times 3.5 \mathrm{~cm}$ and comprised the appendix, which appeared slightly dilated. Microscopically the endocardium showed oedema and an essentially lymphocytic infiltrate which was also present in the myocardial interstitium. There was focal vacuolization and necrosis of myocytes (Fig. 6), sometimes 


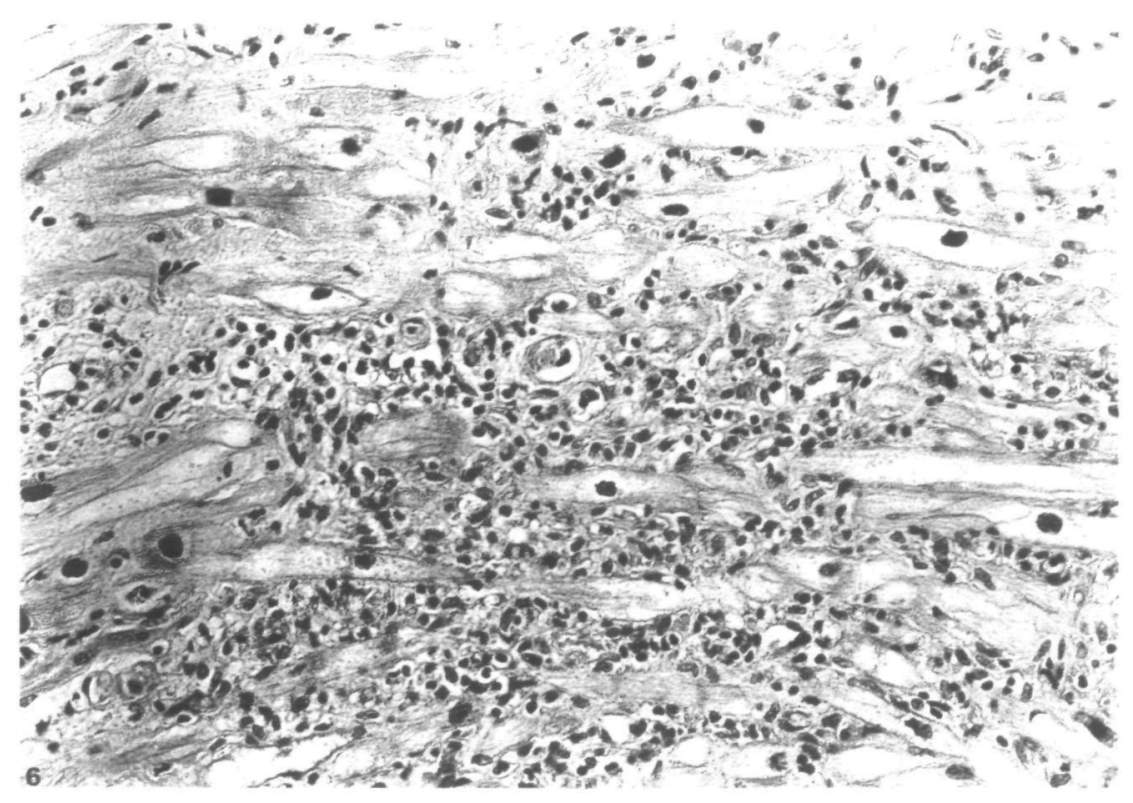

Figure $\sigma$ Focal and confluent lymphocytic infiltrates in the myocardium with vacuolization and focal necrosis of myocytes, some of which show large hyperchromatic nuclei. Haematoxylin and $\operatorname{cosin} \times 250$.

accompanied by small haemorrhages. There was also some degree of interstitial fibrosis. The microscopic findings are consistent with a moderate diffuse lymphocytic myocarditis with some interstitial fibrosis of unknown origin.

\section{Discussion}

Several reports in the literature have discussed the diagnostic, therapeutic and electrophysiologic aspects of ectopic (right) atrial tachycardia ${ }^{[1-3,6.7]}$. However, the probable anatomic substrate for these tachycardias is difficult to investigate. Wyndham et al. ${ }^{[2]}$ described the histologic findings of one patient with ectopic right AT demonstrating mononuclear cell infiltration and increased connective tissue. Olsson et al. ${ }^{[1]}$ described two patients with ectopic AT. In one of those patients three small aneurysms were seen located between the sinus node and right atrial appendage. After excision of this area, sinus rhythm reappeared. Results of histologic examination were not given.

In the two cases described here, no aneurysmal formations were observed. Histologic examination showed a subacute myocarditis in one case and an on-going chronic myocarditis in the other ${ }^{[?]}$.

Biopsies of the right ventricle showed no concomitant ventricular myocarditis, however, proof that the disease was only affecting the right atrium was unavailable. The etiology of the disease is not clear yet. In case 1 serologic investigations for a viral infection have not been done. In case 2 a mild elevation of antibodies to echoviruses was found. In both cases the endocardial catheter mapping showed large areas in the right atrium with abnormal electrical activity. After intra-operative confirmation of these findings and resection of those abnormal zones, the ectopic tachycardia disappeared. We therefore conclude that in some cases with ectopic (right) AT, an isolated myocarditis of the atrium may be the anatomic substrate for the arrhythmia. Careful histologic examination of the resected areas may clarify the pathogenesis of ectopic AT in a subgroup of patients. Further investigations are necessary to clarify whether the management of these patients requires antiinflammatory drug therapy to avoid dissemination of the disease.

\section{References}

[1] Olsson SB, Blomstroem P, Sabel K-G, William-Olsson G. Incessant ectopic atrial tachycardia: successful surgical treatment with regression of dilated cardiomyopathy picture. Am J Cardiol 1984; 53: 1465-6.

[2] Wyndham CRC, Amsdorf MF, Levitsky S et al. Successful surgical excision of focal paroxysmal atrial tachycardia. Circulation 1980; 6: 1365-72. 
[3] Seais AA, Lawrie GM, Magro S et al. Surgical treatment of right atrial focal tachycardia in adults. J Am Coll Cardiol 1988; 11 : 1111-7.

[4] Shenasa M, Fromer M, Faugere $G$ et al. Efficacy and safety of intravenous and oral diltiazem for WolffParkinson-White syndrome. Am J Cardiol 1987; 59: 301-6.

[5] Aretz HT. Myocarditis: the Dallas criteria. Hum Pathol 1987; 18: 619-24.
[6] Mirowski M, Lau SH, Wit AL et al. Ectopic nght atrial rhythms: experimental and clinical data. Am Heart J 1971; 81: 666-76.

[7] Scheinman MM, Basu D, Hollenberg M. Electrophysiologic studies in patients with persistent atrial tachycardia. Circulation 1974; 50: 266-73. 